\title{
Organic chemistry on surfaces: Direct cyclopropanation by dihalocarbene addition to vinyl terminated self-assembled monolayers (SAMs)
}

Malgorzata Adamkiewicz, David O'Hagan* and Georg Hähner ${ }^{*}$

\author{
Full Research Paper \\ Address: \\ EaStCHEM School of Chemistry, University of St. Andrews, St. \\ Andrews, Fife KY16 9ST, UK \\ Email: \\ David O'Hagan* - do1@st-andrews.ac.uk; Georg Hähner* - \\ gh23@st-andrews.ac.uk \\ * Corresponding author \\ Keywords: \\ difluoro-; dichloro-; dibromomethylenecyclopropanes; dihalocarbenes: \\ self-assembled monolayers; surface coating
}

Beilstein J. Org. Chem. 2014, 10, 2897-2902. doi:10.3762/bjoc. 10.307

Received: 11 July 2014

Accepted: 11 November 2014

Published: 05 December 2014

Associate Editor: J. A. Porco Jr.

(C) 2014 Adamkiewicz et al; licensee Beilstein-Institut. License and terms: see end of document.

\begin{abstract}
C11-Vinyl-terminated self-assembled monolayers (SAMs) on silica surfaces are successfully modified in C-C bond forming reactions with dihalocarbenes to generate SAMs, terminated with dihalo- (fluoro, chloro, bromo) cyclopropane motifs with about $30 \%$ surface coverage.
\end{abstract}

\section{Introduction}

Self-assembled monolayers (SAMs) are increasingly being used as a means of surface modification to alter properties in a tuneable manner [1-3]. The major classes of SAMs are those with adsorbed long chain alkyl thiols on gold surfaces/nanoparticles [4,5], or long chain alkylsilanes on silica surfaces [6,7]. Two general approaches are taken to achieve surface modification as illustrated in Figure 1. The first involves incorporating prefunctionalised alkylsilanes/alkylthiols carrying functional groups (FG) to generate the SAM directly, whereas the second approach involves chemical modification of a pre-assembled monolayer carrying reactive groups (RG), as a means to introduce the SAM carrying the FGs [8]. Both approaches present challenges. In the former the desired functionality (FG) requires to be robust and orthogonal in reactivity to the chemistry involved in securing the substrate to the organic film (e.g., FG-Alkyl- $\mathrm{SiCl}_{3}$ and silicon substrate). In the latter chemical modification of the reactive groups of the pre-coated SAM has to be efficient enough such that a reasonable conversion can be obtained, with chemical specificity and lack of surface degradation. In this respect 'click' reactions have become attractive including azide-alkyne cycloadditions $[9,10]$, Diels-Alder reactions [11,12], maleimide-thiol reactions [13], thiol-ene additions [14], and imine/oxime conjugations [15]. In this article we demonstrate that dihalocarbenes can be used to generate dihalocyclopropanes on olefin terminated SAMs.

We recently reported the formation of high quality vinyl-terminated SAMs generated from the vapour phase by adsorption of 


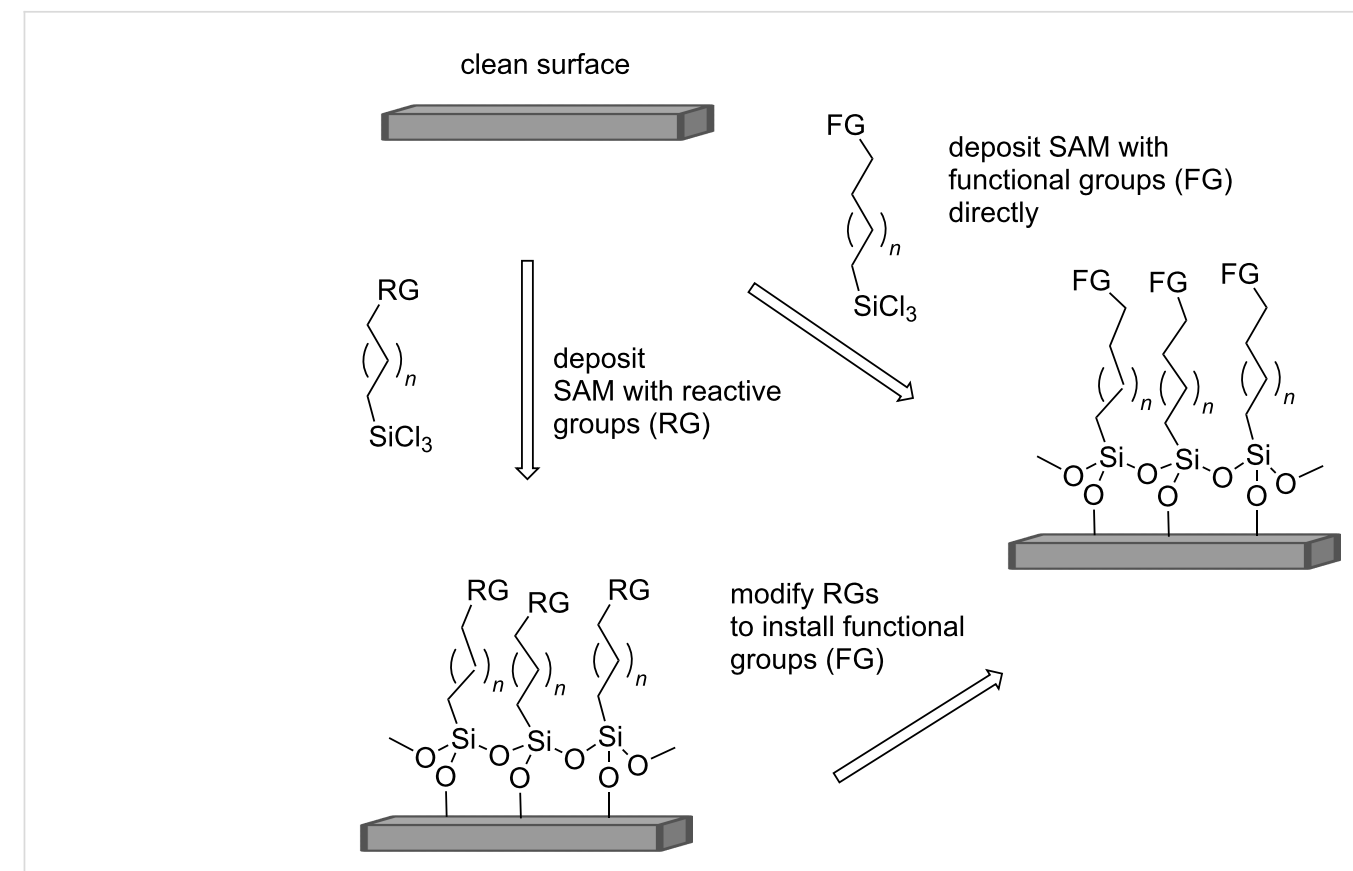

Figure 1: General strategies for incorporating functional groups (FGs) on the surface of self-assembled monolayers (SAMs), illustrated for alkylsilanes onto silica.

octadecyltrichlorosilanes onto silicon wafers [16]. With access to these SAMs it became an objective to explore functional group modification of the vinyl double bond. Carbon-carbon bond formation of vinyl-terminated SAMs has been demonstrated, e.g., through surface modification of radicals generated by $\mathrm{C}-\mathrm{O}$ bond thermolysis [17] and in a more controlled sense via olefin cross metathesis/enyne metathesis [18] of mixed vinyl and acetylenyl-terminated SAMs followed by Diels-Alder modifications of the resultant dienes [19]. We are not aware however of straightforward carbene additions having been explored with olefin-terminated SAMs. The potential for product cyclopropanes offered a modification of limited steric impact, but if suitably substituted may be used to tune surface properties. Therefore dihalocyclopropanes emerged as an attractive controlled modification particularly as the precursor dihalocarbenes are relatively easily generated [20]. In this context we report dihalocyclopropanation of pre-assembled vinyl-terminated SAMs. Three dihalocarbene modifications were explored involving dibromo- $\left(: \mathrm{CBr}_{2}\right)$, dichloro- $\left(: \mathrm{CCl}_{2}\right)$ and difluoro$\left(: \mathrm{CF}_{2}\right)$ carbenes [21-23]. The resultant SAMs were analysed by $\mathrm{X}$-ray photoelectron spectroscopy (XPS), contact angle goniometry, ellipsometry, and atomic force microscopy.

\section{Results and Discussion}

After exposure to carbenes the vinyl-terminated SAMs were characterised by XPS, contact angle measurements and ellipsometry (see Supporting Information File 1). With XPS elements such as silicon, carbon and oxygen were expected in all cases [16]. In each case control reactions were also carried out on the $\mathrm{C}_{18}$-methyl (Me)-terminated SAMs, to ensure that only the vinyl group was responsible for surface reactivity. The resultant XPS analyses are shown for the vinyl-terminated SAMs for each carbene in Figure 2, and directly underneath, the lower traces illustrate the corresponding analyses after exposure of the carbene solutions to the $\mathrm{C}_{18}$-Me-terminated SAMs.

Figure 2a represents a surface after chemical modification with $: \mathrm{CBr}_{2}$ generated from $\mathrm{CHBr}_{3}$. New peaks appeared at binding energies of 71 and $182 \mathrm{eV}$ in all of the samples. These were assigned to $\mathrm{Br} 3 \mathrm{~d}$ and $\mathrm{Br} 3 \mathrm{p}$ signals, respectively [24,25]. Figure $2 \mathrm{~b}$ shows the results obtained from SAMs after modification with : $\mathrm{CCl}_{2}$ generated from $\mathrm{CHCl}_{3}$. New signals at binding energies of 201 and $270 \mathrm{eV}$ were detected. These were assigned to $\mathrm{Cl} 2 \mathrm{p}$ and $\mathrm{Cl} 2 \mathrm{~s}$ [26]. Finally Figure 2c represents the surface after chemical modification with $: \mathrm{CF}_{2}$ generated from $\mathrm{TMSCF}_{3}$. A new signal at binding energy of $688.7 \mathrm{eV}$ was detected and assigned to $\mathrm{F} 1 \mathrm{~s}$, consistent with a $\mathrm{CF}_{2}$ group present on the surface [27].

It was anticipated that gem-dibromo-, gem-dichloro- and gemdifluorocyclopropane-terminated SAMs will be formed, following the usual transformations of these carbenes with double bonds. To add further support to this expectation, model reactions were carried out under each of the reaction conditions with dec-1-ene (1, Scheme 1). All of the cyclopropane products 2a-c were obtained cleanly and in moderate yields (see 
a)

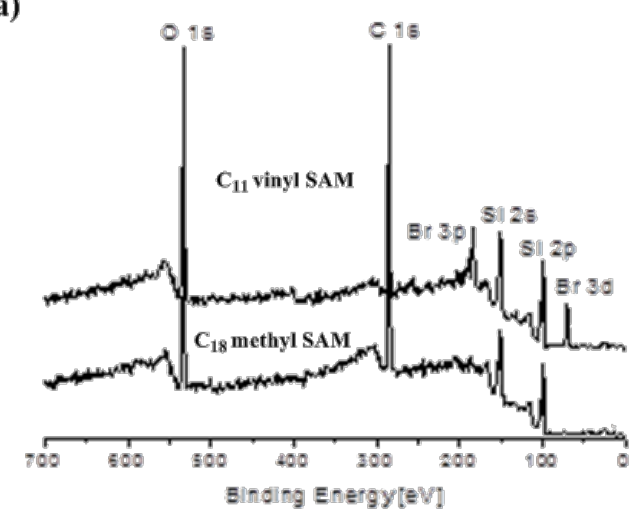

b)

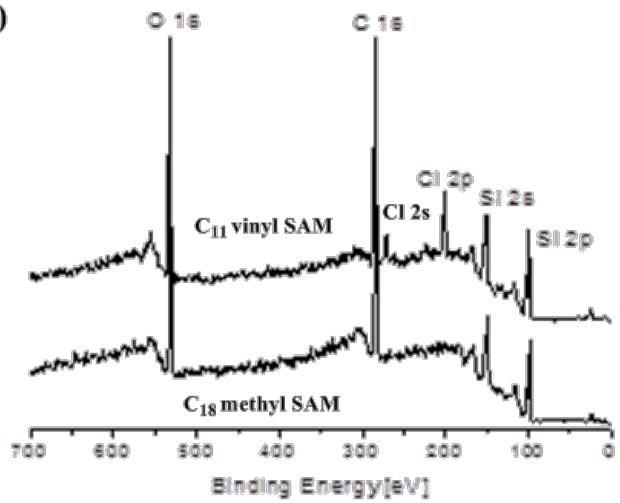

c)

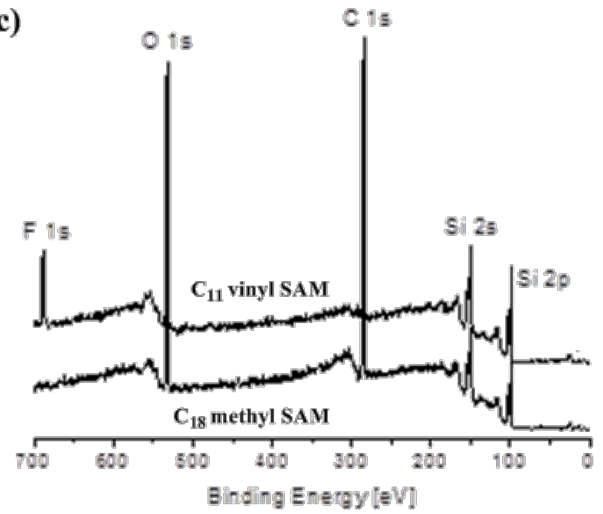

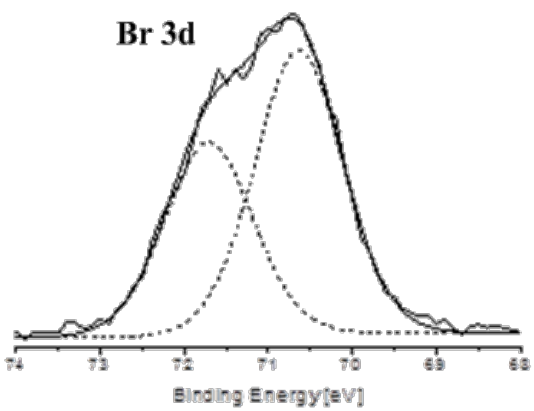
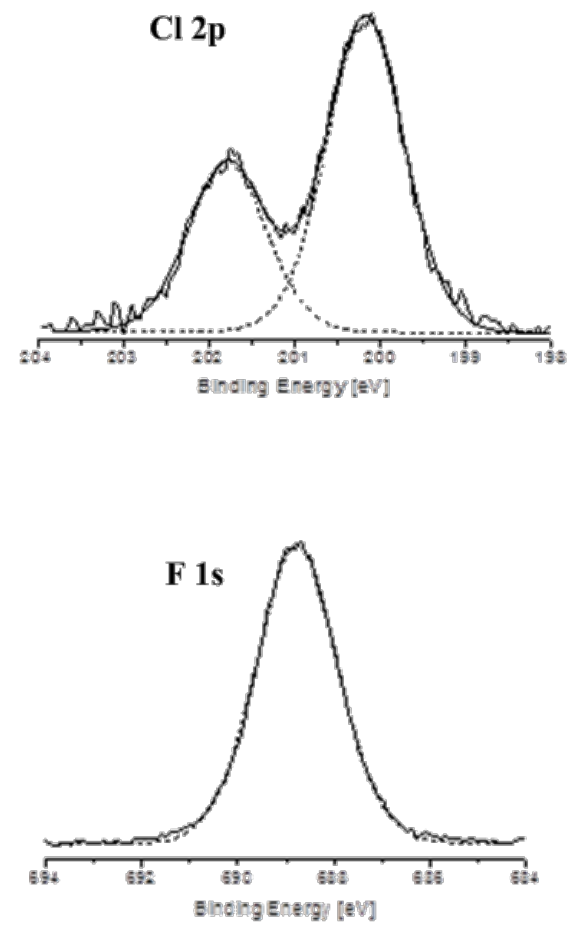

Figure 2: XPS scans after reactions with a) $: \mathrm{CBr}_{2}$; b) : $\mathrm{CCl}_{2}$ and c) $: \mathrm{CF}_{2}$. In each case the upper traces are scans of $\mathrm{C}_{11}$-vinyl SAMs, and the lower traces are $\mathrm{C}_{18}$-Me-terminated SAMs each treated with the relevant carbene reaction solution. The expanded regions on the right hand side, associated with each $\mathrm{C}_{11}$-vinyl SAM spectrum, show the key halide specific XPS signals.

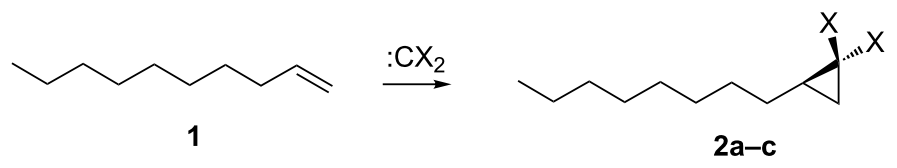

a) $\mathrm{X}=\mathrm{Br}, 54 \%$; b) $\mathrm{X}=\mathrm{Cl}, 49 \%$; c) $\mathrm{X}=\mathrm{F}, 48 \%$

Scheme 1: Model reactions of dec-1-ene (1) with dihalocarbenes in the liquid phase. a) and b) $\mathrm{NaOH}, \mathrm{BTEAC}, \mathrm{CHX}_{3}$, DCM, $8 \mathrm{~h}, 25^{\circ} \mathrm{C}$; c) $\mathrm{TMSCF}_{3}$, $\mathrm{THF}, \mathrm{Nal}, 3 \mathrm{~h}, 65^{\circ} \mathrm{C}$ 
Supporting Information File 1). The results of the model reactions demonstrate that formation of the dihalocyclopropane rings is a relatively clean process for this long chain terminal vinyl substrate. The absence of any side products gives confidence that only dihalocyclopropanes will be formed in the surface reactions.

Turning to the $\mathrm{C}_{11}$-vinyl-terminated SAMs products. In each case the presence of gem-dihalocyclopropane groups on the surface is supported by the ratios of the $\mathrm{C} 1 \mathrm{~s}$ signals to the $\mathrm{Br} 3 \mathrm{~d}, \mathrm{Cl} 2 \mathrm{p}$ or $\mathrm{F} 1 \mathrm{~s}$ signal, respectively. The theoretical and experimental ratios between the carbon and halogen XPS signals are summarised in Table 1 . In all cases the ratios are consistent with a modification coverage of $\sim 30 \%$, with a slightly lower conversion rate in case of $\mathrm{F}$, which might be due to its higher electronegativity and an associated higher repulsion between the terminal groups after cyclopropanation. Conversion rates were determined by correcting the experimental $\mathrm{C} 3 /(\mathrm{C} 1+\mathrm{C} 2)$ ratios from Table 1 with a factor of $d /(\lambda(1-\exp (-d / \lambda))$, where $d$ is the film thickness (determined with ellipsometry) and $\lambda$ the mean free path of the electrons. This accounts for the partial attenuation of the C3 XPS carbon signal. The water contact angles (CAs) of $\mathrm{Br}, \mathrm{Cl}$ and $\mathrm{F}$ carbene treated surfaces were recorded and the $\mathrm{CA}$ values obtained of $80^{\circ}, 85^{\circ}$ and $104^{\circ}$, respectively, are in good agreement with the literature [28-30]. Notably the fluorinated SAM has the largest contact angle as expected, however, the increase and the final contact angle values are clearly lower than that for a pure $\mathrm{CF}_{3}$ terminated film $\left(\sim 118-120^{\circ}\right)$ [31], but this is not surprising given that the halogen functional group density is lower.

Finally AFM images were recorded for the three dihalocyclopropane modified surfaces and they are shown in Figure 3a-c. In all cases the images are smooth and defect free. There was no excess of material observed from reagents after washing, and in each case the RMS surface roughness values did not exceeded $150 \mathrm{pm}$. It is clear that there is no detectable change observed in
Table 1: Assignment of the $C$ 1s XPS signals after treatment of $C_{11}$ vinyl SAMs with the respective dihalocarbene. Theoretical and experimental ratios of the $B r 3 d$ to $C 1 s, C l 2 p$ to $C 1 s$ and $F$ 1s to $C 1 s$ XPS signals of modified $\mathrm{C}_{11}$-vinyl SAMs.
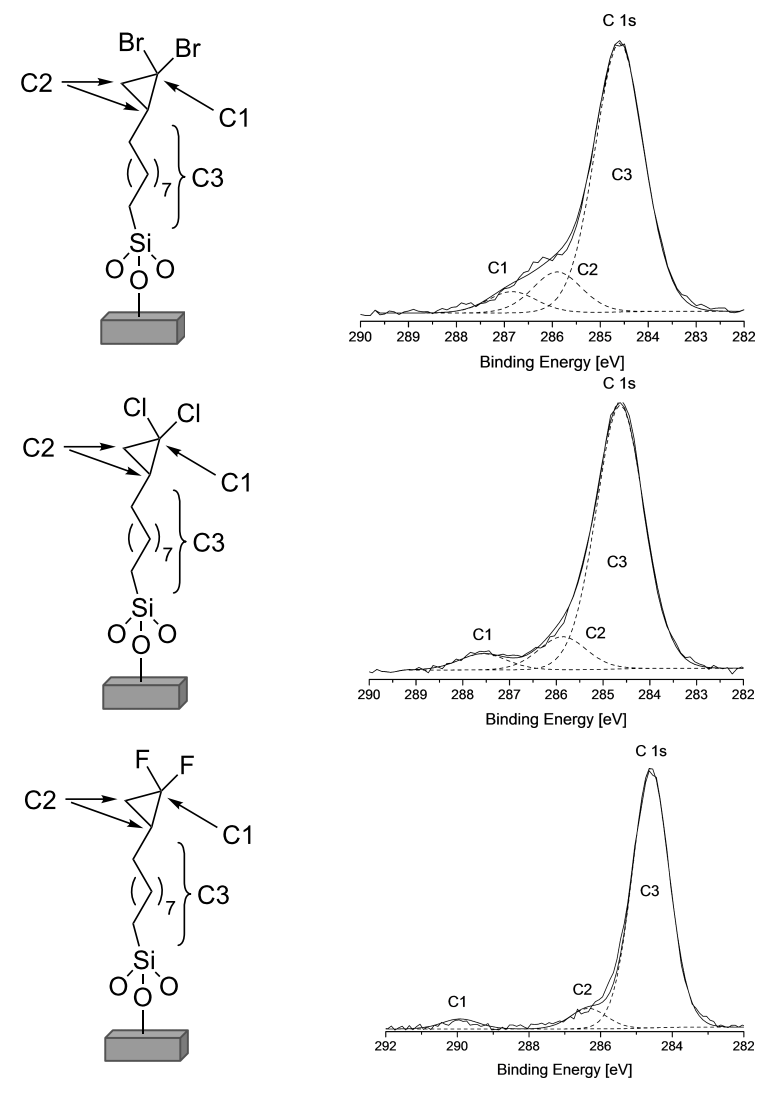

\begin{tabular}{llllll}
\hline \multicolumn{5}{c}{ Ratios } \\
& & $\mathrm{X}: \mathrm{C} 1$ & $\mathrm{X}: \mathrm{C} 2$ & $\mathrm{C} 1: \mathrm{C} 2$ & $\mathrm{C} 3:(\mathrm{C} 1+\mathrm{C} 2)$ \\
\hline Theor. & $: \mathrm{CX}_{2}$ & $2: 1$ & $1: 1$ & $1: 2$ & $3: 1$ \\
Exp. & $: \mathrm{CBr}_{2}$ & $1.9: 1$ & $0.9: 1$ & $1: 2.1$ & $7.6: 1$ \\
Exp. & $: \mathrm{CCl}_{2}$ & $2.2: 1$ & $1.1: 1$ & $1: 2$ & $7.4: 1$ \\
Exp. & $: \mathrm{CF}_{2}$ & $2.1: 1$ & $1: 1$ & $1: 2$ & $8.5: 1$
\end{tabular}

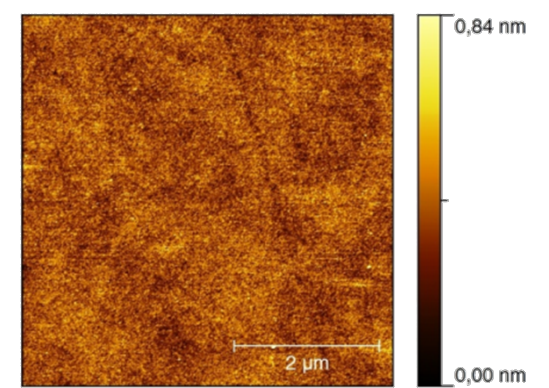

a)

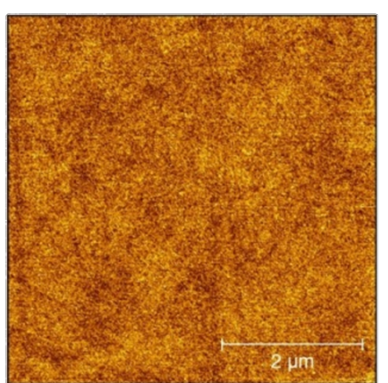

b)

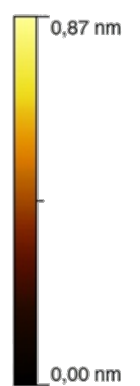

$0,00 \mathrm{~nm}$

c)

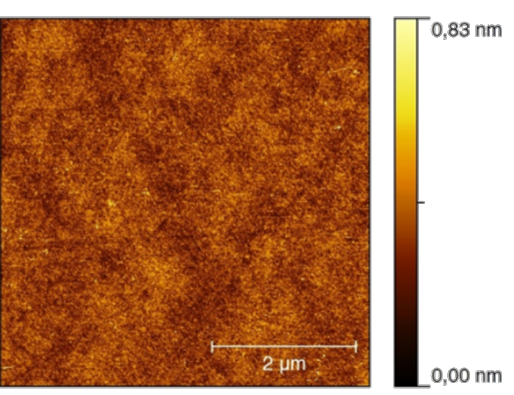

Figure 3: AFM images of $5 \mu \mathrm{m} \times 5 \mu \mathrm{m}$ area of $\mathrm{C}_{11}$-vinyl SAMs modified with a) : $\mathrm{CBr}_{2}$ carbene, RMS $\left.93 \mathrm{pm} ; \mathrm{b}\right): \mathrm{CCl}_{2}$ carbene, $\mathrm{RMS} 101 \mathrm{pm}$; c) $\mathrm{TMSCF}_{3}$, RMS $79 \mathrm{pm}$. 
the film after each modification, and that the films are of good integrity.

An alternative approach, also illustrated in Figure 1 is to prepare SAMs using pure samples of pre-halogenated cyclopropyl chains, with deposition directly onto the surface. This presents the obvious challenge of obtaining highly ordered films after direct deposition. The current approach establishes films of good integrity, which then become chemically modified. There is good evidence that this is less straightforward with functionalised surfactants [32].

\section{Conclusion}

In summary we have been able to demonstrate that vinyl-terminated SAMs can be chemically modified by a range of dihalocarbenes to generate surfaces carrying the corresponding dihalocyclopropane motifs. The reactions demonstrate that these organic chemical transformations, which have been relatively widely used in solution reactions of olefins, can be extended to surface reactions of SAMs. This opens up prospects too of modifying surfaces in this manner with carbenes carrying more elaborate functional groups, and thus a more dramatic change to the surface properties.

\section{Experimental}

Bromoform $\left(\mathrm{CHBr}_{3}\right)$, chloroform $\left(\mathrm{CHCl}_{3}\right)$ and the Ruppert-Prakash reagent $\left(\mathrm{CF}_{3} \mathrm{Si}\left(\mathrm{CH}_{3}\right)_{2}\right)[33,34]$ were used as the carbene precursors for surface modification, with the resultant carbenes generated in solution. For dibromo- and dichlorocarbene generation a solution of $\mathrm{NaOH}$ with $\mathrm{CHBr}_{3}$ or $\mathrm{CHCl}_{3}$ was stirred with a solution of benzyltriethylammonium chloride (BTEAC, $0.1 \mathrm{mmol}$ ) in dichloromethane for $10 \mathrm{~min}$ at $0{ }^{\circ} \mathrm{C}$. Pre-coated silicon wafers $(1 \mathrm{~cm} \times 1.5 \mathrm{~cm})$ with $\mathrm{C}_{11}$-vinyl-terminated SAMs, were immersed in the reaction mixture and the liquids were stirred at room temperature for fixed periods of time (see Supporting Information File 1). SAMs on silicon substrates form stable films [6,7], however, they can be vulnerable to chemical degradation particularly in aqueous base $[28,35,36]$. For this reason the $\mathrm{NaOH}$ concentration and reaction time required to be optimised. The reaction temperature was kept at $25{ }^{\circ} \mathrm{C}$ and the phase-transfer catalyst, benzyltriethylammonium chloride (BTEAC) was chosen to generate the : $\mathrm{CX}_{2}$ carbenes, and minimise exposure of the wafers to the base.

The Ruppert-Prakash reagent $\left(\mathrm{TMSCF}_{3}\right)$ was used to prepare the gem-difluorocyclopropane-terminated SAMs by generating difluorocarbene, following the procedure of Wang et al. [22] for small molecule transformations. This involved stirring a solution of $\mathrm{NaI}$ ( 0.2 equiv) and $\mathrm{TMSCF}_{3}$ in THF $(2 \mathrm{~mL})$, and then immersing the silicon wafers $(1 \mathrm{~cm} \times 1.5 \mathrm{~cm})$ into the reaction mixture at $65{ }^{\circ} \mathrm{C}$ for a fixed period of time (see Supporting Information File 1). The experimental set-up for the surface modification with the three different carbenes is shown in Figure 4. Details of the surface analytical techniques used are given in the Supporting Information File 1.

\section{Supporting Information}

\section{Supporting Information File 1}

Synthesis protocols and additional surface analysis data.

[http://www.beilstein-journals.org/bjoc/content/

supplementary/1860-5397-10-307-S1.pdf]

\section{Acknowledgements}

We thank the Scottish Funding Council for a SPIRIT Studentship (MA) and DO'H thanks the ERC for an Advanced Investigator Grant. Some of the X-ray photoelectron spectra were obtained at the National EPSRC XPS User 's Service (NEXUS) at Newcastle University, UK.

\section{References}

1. Herzer, N.; Haensch, C.; Hoeppener, S.; Schubert, U. S. Langmuir 2010, 26, 8358-8365. doi:10.1021/la9047837

2. Love, J. C.; Estroff, L. A.; Kriebel, J. K.; Nuzzo, R. G.; Whitesides, G. M. Chem. Rev. 2005, 105, 1103-1170. doi:10.1021/cr0300789

3. Ulman, A. Chem. Rev. 1996, 96, 1533-1554. doi:10.1021/cr9502357

4. Häkkinen, H. Nat. Chem. 2012, 4, 443-455. doi:10.1038/nchem.1352
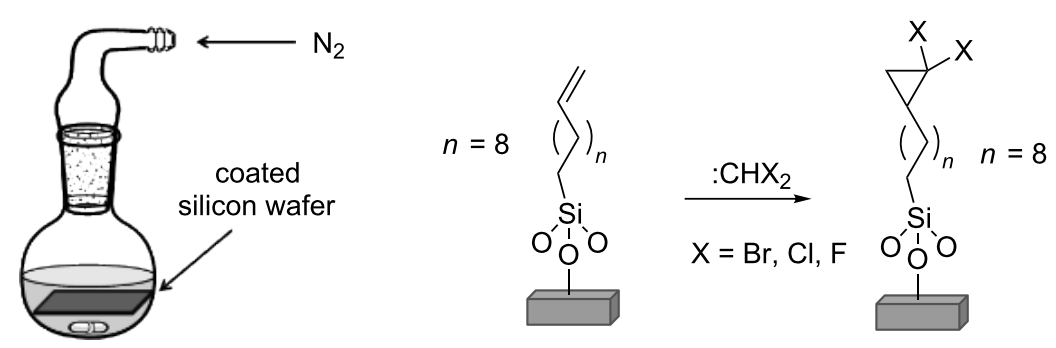

Figure 4: The experimental set-up for the dibromo-, dichloro- and difluorocarbene reactions performed on $\mathrm{C}_{11}$-vinyl $\mathrm{SAMs}_{\text {. }}$ 
5. Vericat, C.; Vela, M. E.; Benitez, G.; Carro, P.; Salvarezza, R. C. Chem. Soc. Rev. 2010, 39, 1805-1834. doi:10.1039/b907301a

6. Haensch, C.; Hoeppener, S.; Schubert, U. S. Chem. Soc. Rev. 2010, 39, 2323-2334. doi:10.1039/b920491a

7. Onclin, S.; Ravoo, B. J.; Reinhoudt, D. N. Angew. Chem., Int. Ed. 2005, 44, 6282-6304. doi:10.1002/anie.200500633

8. Nicosia, C.; Huskens, J. Mater. Horiz. 2014, 1, 32-45. doi:10.1039/c3mh00046j See for a recent excellent overview see.

9. Orski, S. V.; Poloukhtine, A. A.; Arumugam, S.; Mao, L.; Popik, V. V.; Locklin, J. J. Am. Chem. Soc. 2010, 132, 11024-11026. doi:10.1021/ja105066t

10. Collman, J. P.; Devaraj, N. K.; Chidsey, C. E. D. Langmuir 2004, 20, 1051-1053. doi:10.1021/la0362977

11. Arumugam, S.; Popik, V. V. J. Am. Chem. Soc. 2011, 133, 15730-15736. doi:10.1021/ja205652m

12. Yousaf, M. N.; Mrksich, M. J. Am. Chem. Soc. 1999, 121, 4286-4287. doi:10.1021/ja983529t

13. Houseman, B. T.; Gawalt, E. S.; Mrksich, M. Langmuir 2003, 19, 1522-1531. doi:10.1021/la0262304

14. Wendeln, C.; Rinnen, S.; Schulz, C.; Arlinghaus, H. F.; Ravoo, B. J. Langmuir 2010, 26, 15966-15971. doi:10.1021/la102966j

15. Rozkiewicz, D. I.; Kraan, Y.; Werten, M. W. T.; de Wolf, F. A.; Subramaniam, V.; Ravoo, B. J.; Reinhoudt, D. N. Chem. - Eur. J. 2006, 12, 6290-6297. doi:10.1002/chem.200501554

16. Adamkiewicz, M.; O'Hara, T.; O'Hagan, D.; Hähner, G. Thin Solid Films 2012, 520, 6719-6723. doi:10.1016/j.tsf.2012.07.054

17. Siegenthaler, K. O.; Schäfer, A.; Studer, A. J. Am. Chem. Soc. 2007, 129, 5826-5827. doi:10.1021/ja0686716

18. Dutta, S.; Perring, M.; Barrett, S.; Mitchell, M.; Kenis, P. J. A.; Bowden, N. B. Langmuir 2006, 22, 2146-2155. doi:10.1021/la0532196

19. Lee, J. K.; Shi, Y. S.; Lee, J. S.; Kim, Y.-G.; Jung, Y. H.; Oh, E.; Ko, S.-B.; Jung, H.-j.; Kang, P.-S.; Choi, I. S. Langmuir 2005, 21, 10311-10315. doi:10.1021/la051680s

20. Makosza, M. Pure Appl. Chem. 2000, 72, 1399-1403. doi:10.1351/pac200072071399

21. Ziyat, H.; Itto, M. Y. A.; Ali, M. A.; Riahi, A.; Karim, A.; Daran, J.-C. ARKIVOC 2006, xii, 152-160. doi:10.3998/ark.5550190.0007.c18

22. Wang, F.; Luo, T.; Hu, J.; Wang, Y.; Krishnan, H. S.; Jog, P. V.; Ganesh, S. K.; Prakash, G. K. S.; Olah, G. A. Angew. Chem., Int. Ed. 2011, 50, 7153-7157. doi:10.1002/anie.201101691

23. Juliá, S.; Ginebreda, A. Synthesis 1977, 682-683. doi:10.1055/s-1977-24531

24. Balachander, N.; Sukenik, C. N. Langmuir 1990, 6, 1621-1627. doi:10.1021/la00101a001

25. Cohen, Y. S.; Vilan, A.; Ron, I.; Cahen, D. J. Phys. Chem. C 2009, 113, 6174-6181. doi:10.1021/jp9006125

26. Chen, J.-K.; Hsieh, C.-Y.; Huang, C.-F.; Li, P.-M.; Kuo, S.-W.; Chang, F.-C. Macromolecules 2008, 41, 8729-8736. doi:10.1021/ma801127m

27. Ohnishi, S.; Ishida, T.; Yaminsky, V. V.; Christenson, H. K. Langmuir 2000, 16, 2722-2730. doi:10.1021/la991167c

28. Wasserman, S. R.; Tao, Y. T.; Whitesides, G. M. Langmuir 1989, 5, 1074-1087. doi:10.1021/la00088a035

29. Lo, M. K. F.; Gard, M. N.; Goldsmith, B. R.; Garcia-Garibay, M. A.; Monbouquette, H. G. Langmuir 2012, 28, 16156-16166. doi:10.1021/la302880v

30. Motomatsu, M.; Mizutani, W.; Nie, H.-Y.; Tokumoto, H. Thin Solid Films 1996, 281-282, 548-551. doi:10.1016/0040-6090(96)08721-4
31. Colorado, R., Jr.; Lee, T. R. Langmuir 2003, 19, 3288-3296. doi:10.1021/la0263763

32. Fritz, M. C.; Hähner, G.; Spencer, N. D.; Bürli, R.; Vasella, A. Langmuir 1996, 12, 6074-6082. doi:10.1021/la960623j

33. Prakash, G. K. S.; Yudin, A. Chem. Rev. 1997, 97, 757-786. doi:10.1021/cr9408991

34. Prakash, G. K. S.; Krishnamurti, R.; Olah, G. A. J. Am. Chem. Soc. 1989, 111, 393-395. doi:10.1021/ja00183a073

35. Ciampi, S.; Harper, J. B.; Gooding, J. J. Chem. Soc. Rev. 2010, 39 , 2158-2183. doi:10.1039/b923890p

36. Calistri-Yeh, M.; Kramer, E. J.; Sharma, R.; Zhao, W.; Rafailovich, M. H.; Sokolov, J.; Brock, J. D. Langmuir 1996, 12, 2747-2755. doi:10.1021/la950518u

\section{License and Terms}

This is an Open Access article under the terms of the Creative Commons Attribution License

(http://creativecommons.org/licenses/by/2.0), which permits unrestricted use, distribution, and reproduction in any medium, provided the original work is properly cited.

The license is subject to the Beilstein Journal of Organic Chemistry terms and conditions: (http://www.beilstein-journals.org/bjoc)

The definitive version of this article is the electronic one which can be found at: doi:10.3762/bjoc. 10.307 